\title{
Oligospermic infertility associated with an androgen receptor mutation that disrupts interdomain and coactivator (TIF2) interactions
}

\author{
Farid J. Ghadessy, ${ }^{1}$ Joyce Lim, ${ }^{1}$ Abdullah A.R. Abdullah, ${ }^{2,3}$ Valerie Panet-Raymond, ${ }^{2}$ \\ Chee Keong Choo, ${ }^{1}$ Rose Lumbroso, ${ }^{2}$ Thein G. Tut, ${ }^{1}$ Bruce Gottlieb, ${ }^{2}$ \\ Leonard Pinsky, ${ }^{2,3,4,5,6}$ Mark A. Trifiro, ${ }^{2,4}$ and Eu Leong Yong ${ }^{1}$
}

${ }^{1}$ Department of Obstetrics and Gynaecology, National University of Singapore, Republic of Singapore 119074

${ }^{2}$ Lady Davis Institute for Medical Research, Sir Mortimer B. Davis-Jewish General Hospital,

${ }^{3}$ Department of Biology,

${ }^{4}$ Department of Medicine,

${ }^{5}$ Department of Pediatrics, and

${ }^{6}$ Department of Human Genetics, McGill University, Montreal H3T 1E2, Quebec, Canada

Address correspondence to: Eu Leong Yong, Department of Obstetrics and Gynaecology, National University Hospital, Lower Kent Ridge Road, Republic of Singapore 119074. Fax: 65-779-4753; E-mail: obgyel@nus.edu.sg.

Farid J. Ghadessy, Joyce Lim, and Abdullah A.R. Abdullah contributed equally to this work.

Received for publication June 15, 1998, and accepted in revised form April 27, 1999.

Structural changes in the androgen receptor (AR) are one of the causes of defective spermatogenesis. We screened the $A R$ gene of 173 infertile men with impaired spermatogenesis and identified 3 of them, unrelated, who each had a single adenine $\rightarrow$ guanine transition that changed codon 886 in exon 8 from methionine to valine. This mutation was significantly associated with the severely oligospermic phenotype and was not detected in 400 control $A R$ alleles. Despite the location of this substitution in the ligand-binding domain (LBD) of the AR, neither the genital skin fibroblasts of the subjects nor transfected cell types expressing the mutant receptor had any androgen-binding abnormality. However, the mutant receptor had a consistently (approximately 50\%) reduced capacity to transactivate each of 2 different androgen-inducible reporter genes in 3 different cell lines. Deficient transactivation correlated with reduced binding of mutant AR complexes to androgen response elements. Coexpression of AR domain fragments in mammalian and yeast two-hybrid studies suggests that the mutation disrupts interactions of the $\mathrm{LBD}$ with another $\mathrm{LBD}$, with the $\mathrm{NH}_{2}$-terminal transactivation domain, and with the transcriptional intermediary factor TIF2. These data suggest that a functional element centered around M886 has a role, not for ligand binding, but for interdomain and coactivator interactions culminating in the formation of a normal transcription complex.

J. Clin. Invest. 103:1517-1525 (1999).

\section{Introduction}

Infertility affects approximately $10-15 \%$ of all couples (1). Spermatogenesis is at fault in about half of them, but its cause is often covert. Androgens are required for normal spermatogenesis; however, most infertile men with impaired spermatogenesis have normal serum androgen levels. Therefore, attention has turned to the androgen response apparatus, particularly the androgen receptor (AR). Mutation of the X-linked $A R$ gene causes a wide range of clinical androgen insensitivity: complete, when the external genitalia are female; partial, when they are sufficiently ambiguous to require corrective surgery; and mild, when they are phenotypically male. Three surveys of men with idiopathic infertility have yielded widely disparate frequencies of androgen-binding abnormalities in cultured genital skin fibroblasts (2-4). Genetic defects of the $A R$ that cause mild androgen insensitivity with impaired or preserved spermatogenesis (5-7) are of particular interest because they may illuminate the fine structure-function attributes of the AR that permit differential regulation of androgen-inducible genes. To this end, we screened a large group of infertile men with defective spermatogenesis for abnormalities in the coding segments of their $A R$ genes. We found 3 unrelated subjects with the same missense substitution in the COOH-terminal portion of the ligand-binding domain (LBD) of the AR. One shaves infrequently; another has low-grade but persistent postpubertal gynecomastia. Unexpectedly, this novel mutation does not affect the ligand-binding characteristics of the AR; rather, it reduces the transactivational competence of the AR by impairing interactions between the receptor domains, binding to androgen response elements (AREs), and function of the steroid receptor coactivator TIF2.

\section{Methods}

The study population. Our patients presented to infertility clinics because they could not conceive for at least 2 years. None was referred with the problem of sexual ambiguity. Two or more sperm samples were collected at least 3 months apart after 3 - to 5-day abstinence and were assessed according to World Health Organization criteria (8). Patients with abnormal sperm analyses due to obstruction of the genital tract, hypopituitarism, 
hyperprolactinemia, or markedly raised follicle-stimulating hormone were excluded. Of the remaining 173 patients, 33 had azoospermia, 84 were severely oligospermic (less than 5 million/mL), 36 were moderately oligospermic (5-20 million/mL), and 20 had only abnormal sperm motility. Control male subjects $(n=100)$ of proved fertility, no previous infertility history or treatment, and without any genetic disease were recruited from the contraceptive clinic. A further 205 (110 men and 95 women) healthy subjects were also screened to determine whether $A R$ allelic variations exist in the general population.

The mutant subjects CML presented at 31 years of age after 4 years of infertility. He shaves infrequently, approximately once a week. Right-sided cryptorchidism was corrected at 7 years of age. Testicular volume was $10 \mathrm{~mL}$ bilaterally, and his sperm count was approximately 0.5 million $/ \mathrm{mL}$. $\mathrm{KLH}$ presented at 40 years of age after 7 years of infertility. He had Tanner grade 2 persistent postpubertal gynecomastia. Testicular volume was $6 \mathrm{~mL}$ bilaterally, with sperm counts around 0.3 million/mL. Secondary sexual development was otherwise normal in both subjects. The third subject, EHS, had a sperm count of 0.7 million $/ \mathrm{mL}$. All 3 patients had normal male karyotypes and normal serum levels of gonadotropins, androgens, sex hormone-binding globulin, prolactin, and estradiol.

Mutational screening with single-stranded conformational polymorphism. DNA was extracted from peripheral blood. Coding segments and flanking intronic sequences of exons 2-8 of all 173 patients were examined by single-stranded conformational polymorphism (SSCP) (9). DNA fragments that exhibited differential mobilities were sequenced. Exons 2-8 of the patients (CML, KLH, EHS) with mutant alleles were reexamined by SSCP to exclude any coexisting mutations that might have been missed on initial screening. Most of exon 1 of the mutant subjects was screened by SSCP. The 2 highly polymorphic trinucleotide (CAG and GGC) tracts were directly sequenced.

In vivo androgen-binding properties of $A R$. Fibroblast cultures were obtained from skin biopsies of subjects and from normal controls. The androgen-binding properties of the AR were determined according to standard techniques (10). Finasteride $\left(10^{-7}\right.$ $\mathrm{M})$ was added to the cultures to inhibit endogenous $5 \alpha$-reductase activity that might degrade androgens. Scatchard analysis was performed by plotting specifically bound hormone versus the bound/free ratio. $K_{\mathrm{d}}$ was determined from the negative slope of the graph. $\mathrm{B}_{\max }$ was obtained from the intercept of the line on the $x$ axis. Thermolability was examined by comparing the binding properties at $32^{\circ} \mathrm{C}$ or $37^{\circ} \mathrm{C}$, and $42^{\circ} \mathrm{C}$. A reduction of $B_{\max }$ of more than $40 \%$ defined thermolability.

Chase experiments. To determine $K_{\mathrm{d}}$, the rate constant of dissociation, cell monolayers were preincubated with the radiolabeled androgens, and the proportion of labeled hormone still specifically bound after exposure to excess unlabeled hormone was determined at defined time intervals.

\section{Plasmids}

M886V AR. The mutation in our subjects was recreated in a cDNA fragment by site-directed mutagenesis (6) and then substituted into the homologous section of an AR expression vector, pSVhARo. Transactivation domain (TAD) and LBD AR fragments were formed from AR cDNA by excising fragments bounded by the unique restriction sites $K p n I / E c o$ RI and NheI/KpnI, respectively.

Mammalian two-bybrid. The pGAL4DBD-LBD (CLONTECH Laboratories Inc., Palo Alto, CA) was prepared by amplifying cDNA encoding the AR LBD (exons 4-8) and ligating in-frame into the SmaI/HindIII site of pM containing GAL4 DNA-binding domain. The PVP16AD-ARTAD was made by restricting pSVhARo with EagI and HindIII to release the fragment encoding amino acids $14-565$ of the AR. The $5^{\prime}$ end of this fragment a

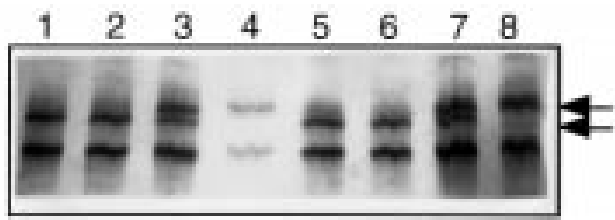

b

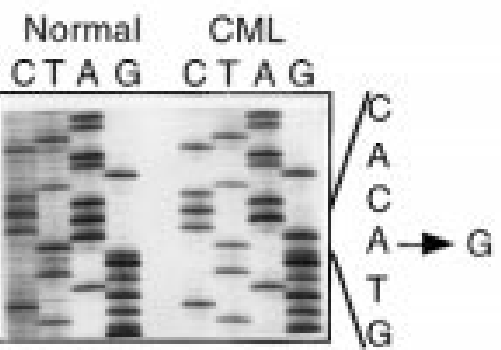

c
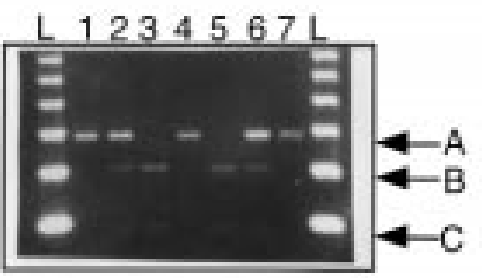

\section{Figure 1}

(a) SSCP analyses of family members of 2 probands. Exon 8 fragments were amplified from genomic DNA and electrophoresed on a PAGE gel to display SSCP mobility shifts. The probands, CML (lanes 2 and 6 ) and KLH (lanes 1 and 5), have a mutant DNA strand (lower arrow) that migrates faster than the WT allele (upper arrow) from the unaffected siblings (sister of CML, lane 4; brother of $\mathrm{KLH}$, lane 8). Mothers of CML (lane 3 ) and KLH (lane 7) have both mutant and WT alleles, indicating that both women are carriers. (b) Sequencing autoradiogram of a portion of $A R$ exon 8 from patient CML compared with the normal. Three patients had the same $A \rightarrow G$ substitution. (c) Restriction analyses of family members of 2 patients (CML, KLH). Exon 8 fragments were amplified from genomic DNA and restricted with $B b r P 1$. The M $886 \mathrm{~V}$ mutation creates a new $B b r P 1$ site such that enzymatic digestion results in 2 fragments (B and $\mathbf{C}$ ) in the probands (lane 3, CML; lane 5, KLH), whereas normal alleles (lane 1, sister of CML; lane 4, normal fertile man; lane 7, brother of KLH) have only 1 fragment (A), measuring 347 bp. Mothers of CML (lane 2) and KLH (lane 6) display all 3 fragments, indicating their heterozygous status. Outer lanes are 123-bp DNA ladders (L).

was then ligated to a synthetic linker encoding the first 13 amino acids of ARTAD, and the resultant fragment encoding the entire ARTAD was cloned in-frame with the VP16 activation domain using pVP16 vector. Plasmid PVP16AD-TIF2 was constructed by a double digest of PSG5-TIF2 with HindIII/XbaI followed by ligating in-frame to pVP16. The $(17 \mathrm{~m})_{5}$-E1bTATA-Luc reporter vector was obtained by amplifying the 5 GAL4 binding sites and the adenovirus E1b minimal promoter of PG5CAT and ligating upstream of the luciferase gene in PGL-basic (Promega Corp., Madison, Wisconsin, USA) vector.

Yeast two-bybrid. Wild-type (WT) or M886V LBD fragments including (amino acids 502-919) or excluding (amino acids 659-919) the DNA-binding domain (DBD) were generated by double digesting the full-length $\mathrm{AR}$ vector with $K p n \mathrm{I} / B a m \mathrm{HI}$ or $P v u \mathrm{I} / B a m \mathrm{HI}$, respectively. The LBD fragments were cloned into vectors pAS2-1 and pACT2, which contain the GAL4 DNA-binding domain (amino acids 1-147) and the GAL4 transactivation domain (amino acids 768-881), respectively, to generate GAL4-AR hybrid constructs. Full-length AR constructs were made by excising the AR from pSVhARo.BHEX using SmaI/BamHI and then ligating the resulting fragment to 

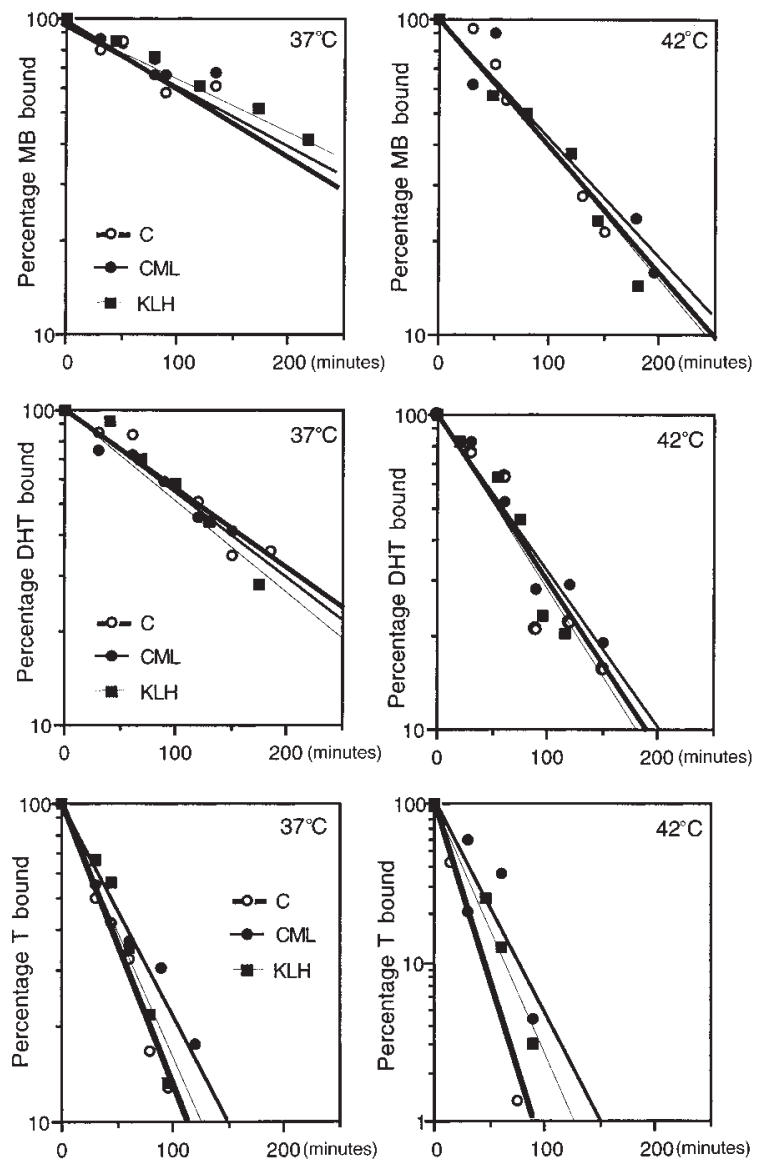

Figure 2

Dissociation kinetics of ARs in genital skin fibroblasts. Normal (C, open circles and bold lines) and mutant (CML, filled circles and normal lines; $\mathrm{KLH}$, filled squares and thin lines) fibroblast monolayers were exposed to $2 \mathrm{nM}\left[{ }^{3} \mathrm{H}\right] \mathrm{MB}$ (top), $3 \mathrm{nM}\left[{ }^{3} \mathrm{H}\right] \mathrm{DHT}$ (middle), or $3 \mathrm{nM}\left[{ }^{3} \mathrm{H}\right] \mathrm{T}$ (bottom) at $37^{\circ} \mathrm{C}$ (left) or $42^{\circ} \mathrm{C}$ (right) for 2 hours. The radiolabeled medium was discarded and replaced with one containing 200 -fold excess unlabeled androgen; replicate samples were removed at the indicated times and assayed for $\left[{ }^{3} \mathrm{H}\right]$ androgen that was still receptor bound. Each data point, the mean of 4 replicates, is expressed as a percentage of maximum binding at time 0 . Vertical axes are on the same logarithmic scale, except for bottom right panel.

pAS2-1 and PACT2 that had been similarly digested. All constructs were sequenced to confirm the fidelity of the enzymatic manipulations.

Mammalian cell culture and transient transfection. Mutant and WT plasmids were transfected into COS-7, CV-1, or HeLa cells using lipofection technique (11). pCMV- $\beta$ Gal was used to assess transfection efficiency. In some replicates, radiolabeled mibolerone $(\mathrm{MB})$ was added to the culture medium and specific MB-binding activity was determined (6). Transactivation activity was measured in relative light units (RLU) and normalized to protein content and transfection efficiency.

Immunoblot analyses. Immunoblot analyses were used to study the effect of the mutation on AR protein production. The rabbit polyclonal antibody PG-21, which recognizes the first 21 $\mathrm{NH}_{2}$-terminal amino acids of the human $\mathrm{AR}$, was used to detect AR protein (12). Mouse mAb SC510 (Santa Cruz Biotechnology Inc., Santa Cruz, California, USA) was used to locate GAL4DBD fusion proteins. Protein-antibody complexes were subsequently viewed by enhanced chemiluminescence (11).
DNA mobility gel shift assays and quantitation of androgen-AR complexes bound to AREs

DNA mobility gel shift assays. COS-7 cells were transfected with WT or mutant AR plasmids, harvested in extraction buffer $(20$ mM HEPES [pH 7.9], 20\% glycerol, $100 \mathrm{mM} \mathrm{KCl,} 0.2 \mathrm{mM}$ EDTA) containing protease inhibitors (phenylmethylsulfonylfluoride, leupeptin, aprotinin), lysed by 3 freeze/thaw cycles to release AR. A consensus synthetic ARE (5'-CTAGAAGTCTGGTACAGGGTGTTCTTTTTGCA-3'), served as specific-binding DNA (13). Two nonspecific competitor oligonucleotides were used. The first incorporates a WT estrogen response element (ERE) from the promoter of the Xenopus vitellogenin A2 gene: 5'-GTCCAAAGTCAGGTCACAGTGACCTGATCAAAGTT-3'; the second incorporates a transcription factor Oct2A response element: $5^{\prime}$-GTACGGAGTATCCAGCTCCGTAGCATGCAAATCCTCTGG-3'. T4 polynucleotide kinase and $\left[\gamma_{-32}{ }^{32}\right]$ dATP were used to label the oligonucleotides. Binding reactions containing $5 \mu \mathrm{g}$ cell extract, $60 \mu \mathrm{g}$ BSA, $10 \%$ glycerol, $2 \mathrm{mM}$ DTT, $2 \mu \mathrm{g}$ poly(dI-dC), $0.2 \mathrm{mM}$ EDTA, and $20 \mathrm{mM}$ HEPES ( $\mathrm{pH} 7.9$ ) in a total volume of $20 \mu \mathrm{L}$ were preincubated on ice for 20 minutes. Some reactions contained a 25 -fold excess of unlabeled oligonucleotide as competitor DNA. Labeled oligonucleotides $(\sim 0.3 \mathrm{ng}, 10,000 \mathrm{cpm})$ were added and incubation carried out a further 20 minutes at room temperature. AR-DNA complexes were resolved by electrophoresis on $5 \%$ PAGE gels and autoradiographed.

Quantitation of androgen-AR complexes bound to AREs. AR expressed in COS- 1 cells was exposed to $3 \mathrm{nM}\left[{ }^{3} \mathrm{H}\right] \mathrm{MB}$ and harvested, and aliquots were measured for radioactivity. Each assay mixture contained $10 \mu \mathrm{g}$ of poly(dI-dC), a sample of charcoal-treated supernate with 50,000 dpm of MB-receptor complexes, and binding buffer to a final volume of $500 \mu \mathrm{L}$. Then we added $150 \mathrm{pmol}$ of $3^{\prime}$-biotinylated doublestranded oligonucleotide sequence of the synthetic ARE (5'-CTAGAAGTCTGGTACAGGGTGTTCTTTTTGCA-biotin), or MMTV-ARE (5'-TATGGTTACAAACTGTTCTTAAAAbiotin) and $50 \mu \mathrm{L}$ of streptavidin-agarose beads, before continuing incubation for 2 hours. The beads were collected by centrifugation, washed three times, and bound radioactivity was measured by liquid scintillation. Assay mixtures lacking an ARE bound negligible amounts of radioactivity. Each data point was the mean of duplicate experiments calculated as a percentage of binding observed with WT AR $(14,15)$.

Yeast two-hybrid assay for LBD-LBD interactions. Hybrid GAL4AR proteins were expressed in Saccharomyces cerevisiae Y190 containing integrated GAL4 binding sites upstream of the $U A S_{G A L 1}$-lac $Z$ reporter gene. Interaction between hybrid proteins results in a transcriptionally active complex inducing $\beta$ galactosidase activity. Yeast transformation was performed using the lithium acetate method according to protocols. Yeast were grown in standard YEDP medium or an appropriate selective medium in the presence or absence of $1 \mathrm{M}$ methyltrienolone (MT). Yeast were harvested, and liquid $\beta$-galactosidase assays were performed using $o$-nitrophenyl- $\beta$-D-galactoside (ONPG) as a substrate according to standard protocols with the following modification: the yeast were permeabilized by incubation in a $0.2 \%$ sodium lauryl sarcosinate $\mathrm{Z}$ buffer solution $\left(60 \mathrm{nM} \mathrm{Na}_{2} \mathrm{HPO}_{4}, 40 \mathrm{nM} \mathrm{NaH} \mathrm{PO}_{4}, 10 \mathrm{mM} \mathrm{KCl}, 1\right.$ $\mathrm{mM} \mathrm{Mg}_{2} \mathrm{SO}_{4}, 50 \mathrm{mM} \beta$-mercaptoethanol) instead of freezethawing before the addition of ONPG.

\section{Results}

Mutations detected in the AR of 3 unrelated oligospermic patients. Three unrelated patients - CML, KLH, and EHS - showed differential migration of exon 8 PCR fragments when screened by SSCP. Two subjects, CML and 
a

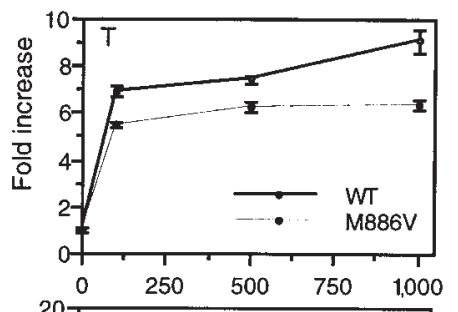

b

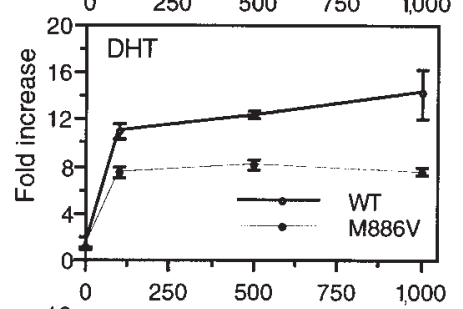

c

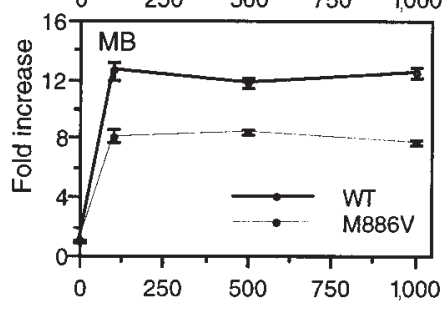

Figure 3

Transactivation activity of M886V AR with high doses of androgens. WT (open circles and solid lines) or mutant (filled circles and dotted lines) receptors were transiently expressed in COS-7 cells and exposed to increasing doses (nM) of T (a), DHT (b), or MB (c). Transactivation activity was expressed as fold increase in luciferase activity compared with cells not exposed to androgen. $\beta$-galactosidase activity and protein content were used to normalize for transfection efficiency and cell numbers, respectively. Each data point represents the mean \pm SE of 4 replicates.

$\mathrm{KLH}$, inherited the mutation from their mothers, who were heterozygous carriers (Figure 1a). The suspected fragments were sequenced, and all 3 patients had the same mutation in exon 8 involving amino acid 886, an $A \rightarrow G$ transition resulting in substitution of valine for methionine (Figure 1b). This mutation resulted in the creation of a new $B b r \mathrm{P} 1$ restriction site (Figure 1c). All 3 subjects were near-azoospermic, with repeated sperm counts less than 1 million/mL. CML had 23 codons in each exon 1 polymorphic trinucleotide repeat tract, whereas KLH had 21 glutamine and 24 glycine codons, confirming that the 2 subjects were not genetically related. No coexisting mutations were detected in $A R$ exons 1-8 of the 3 patients on SSCP analyses, nor in exon 8 PCR fragments of $400 A R$ alleles from healthy controls. The presence of 3 mutations in our 173 infertile patients and none in 400 control alleles makes it unlikely that M886V exists in the general population (Fisher's exact test, $P=0.027)$. On the other hand, as the mutations occur only in a subset of infertile men with severe oligospermia $(n=84)$, it is more likely $(P=0.005)$ that $\mathrm{M} 886 \mathrm{~V}$ is significantly associated with the phenotype of severe oligospermia.

M886V in the LBD bad no effect on androgen-binding characteristics. The $K_{\mathrm{d}}$ for dihydrotestosterone (DHT) was 0.31 and $0.52 \mathrm{nM}$, and the $\mathrm{B}_{\max }$ was 36 and $37 \mathrm{fmol} \mathrm{DHT} / \mathrm{mg}$ protein for $\mathrm{CML}$ and $\mathrm{KLH}$, respectively, at $37^{\circ} \mathrm{C}$ for fibroblast monolayers cultured from scrotal skin biopsies (normal: $K_{\mathrm{d}}, 0.3-0.6 \mathrm{nM} ; \mathrm{B}_{\max }, 26-43 \mathrm{fmol} / \mathrm{mg}$ protein). Similarly, there were no significant differences in affinity constants for testosterone (T), the $K_{\mathrm{d}}$ for CML being $1.28 \mathrm{nM}$ (normal: $\sim 1.8 \mathrm{nM}$ ). With MB, $K_{\mathrm{d}}$ for KLH fibroblasts was $0.1 \mathrm{nM}$ and $\mathrm{B}_{\max }$ was $52 \mathrm{fmol} / \mathrm{mg}$ protein; with MT (R1881), $K_{\mathrm{d}}$ was $0.12 \mathrm{nM}$ and $\mathrm{B}_{\max }$ was $52 \mathrm{fmol} / \mathrm{mg}$ protein (normal: $K_{\mathrm{d}}, 0.1-0.3 \mathrm{nM} ; \mathrm{B}_{\max }, 15-50 \mathrm{fmol} / \mathrm{mg}$ protein). When exposed to DHT at $42^{\circ} \mathrm{C}$, the $K_{\mathrm{d}}$ and $\mathrm{B}_{\max }$ values of AR in CML fibroblasts $(0.27 \mathrm{nM}$ and $35 \mathrm{fmol}$ $\mathrm{DHT} / \mathrm{mg}$ protein, respectively) were no different from the corresponding values at $37^{\circ} \mathrm{C}$, indicating the absence of thermolability. The experiment was repeated using MB and MT, with similar results (data not shown). Prolonged labeling for 15 hours at $37^{\circ} \mathrm{C}$ with $2 \mathrm{nM} \mathrm{MT}$, or MB, followed by a switch to $41^{\circ} \mathrm{C}$ or $42^{\circ} \mathrm{C}$ for up to 6 hours in the presence of $100 \mathrm{mM}$ cycloheximide, failed to demonstrate any difference between mutant and normal cells at the higher temperatures. Chase experiments were performed to examine the dissociation kinetics of mutant AR complexes. When chased at $37^{\circ} \mathrm{C}$ or $42^{\circ} \mathrm{C}$, the dissociation rates for $\mathrm{T}, \mathrm{DHT}$, and $\mathrm{MB}$ were similar for both normal and mutant fibroblasts (Figure 2). After an 18hour incubation with $\mathrm{T}$, the remaining $\mathrm{AR}$ complexes dissociated linearly with a $k$ value of $5\left(10^{-3} / \mathrm{min}\right)$ at $37^{\circ} \mathrm{C}$. This value is typical of DHT-receptor complexes and indicates normal conversion of T to DHT. The AR content of CML fibroblasts rose from 48 to $104 \mathrm{fmol} / \mathrm{mg}$ protein when incubated with MB for 2 and 20 hours, respectively, indicating normal receptor upregulation. Normally, a doubling or greater of specific androgenbinding activity is observed after overnight incubation with androgen, presumably because of AR stabilization by ligand. Likewise, the affinity and dissociation characteristics of androgens bound to mutant receptors expressed in COS-7 cells were no different from the WT (data not shown). In aggregate, these experiments, independently replicated in 2 laboratories, indicate that the M886V mutation of the LBD did not change any androgen-binding properties of the receptor.

Impaired transactivation capacity of the mutant receptor. The mutant receptor, however, had only $50-70 \%$ of the transactivation capacity of the WT receptor in COS-7 cells when exposed to various concentrations of T, DHT, and MB. The ARs encoded by the normal and mutant plasmids demonstrated dose-dependent increases in transactivation capacity with $\mathrm{T}$, but at all doses from 10 to $300 \mathrm{nM}$, the transcriptional capacity of M886V AR was only about half that of the WT (data not shown). The transactivational defect persisted even with doses of androgens up to $1 \mu \mathrm{M}$ (Figure 3). Thus, the mutant AR had a modest but consistent reduction in its transactivation capability compared with that of the WT, for all 3 androgens, in repeated experiments. Using a cell line (CV1) that does not express the SV-40 antigen, the transactivation defect of the mutant AR was even more evident. Thus, when 50,100, and 200 ng of AR cDNA were used in the transfections, the WT gave 3.1-, 11.1-, and 3.4-fold higher transactivation activity, respectively, compared with the mutant (Figure 4a). Immunoblot analyses showed that AR protein levels were equivalent for mutant and WT transfections, at all 3 cDNA doses (Fig- 
a

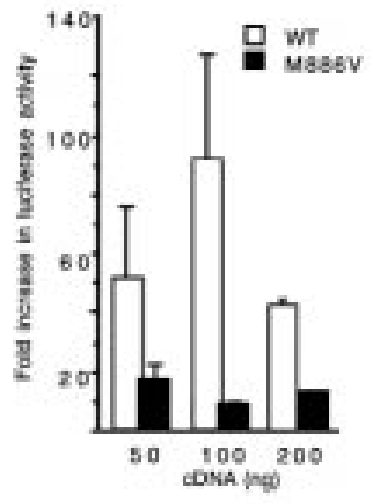

b

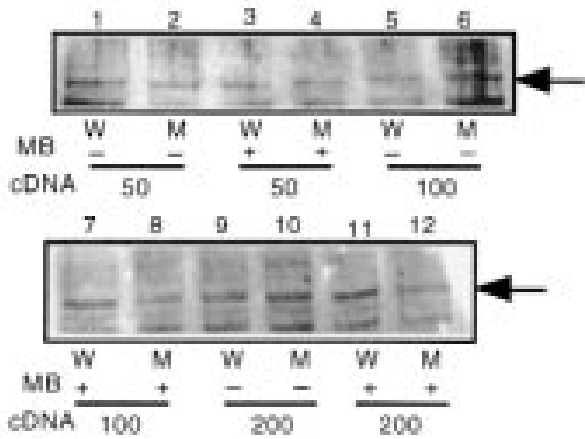

Figure 4

(a) Transactivation activity of M886V AR with increasing doses of AR cDNA. CV1 cells were cotransfected with the indicated amounts of WT or M886V AR cDNA and the reporter plasmid pMAM-LUC. Each data point, the mean of triplicates, represents the fold increase in luciferase activity of cells exposed to $30 \mathrm{nM} \mathrm{MB}$ compared with those without androgen. Bars are \pm SE. (b) Immunoblot of WT (W) or mutant (M) receptors. CV1 cell extracts (10 $\mu \mathrm{g}$ total protein each) depicted in a were electrophoresed on an SDS-PAGE gel, and AR protein identified with a specific antibody (PG-21). Films were overexposed to enhance signal for the low-abundance AR protein.

ure $4 \mathrm{~b})$. Androgen-binding activity of the mutant and WT AR proteins in COS cells was also similar (data not shown), confirming that the transactivation defect of $\mathrm{M} 886 \mathrm{~V}$ was not due to changes in AR protein levels.

Impaired binding of M886V ligand-AR complexes to AREs. Both WT and mutant receptors displayed an increase in ARE binding with the higher hormone dose (Figure 5a, compare lane 1 with 2 , and lane 3 with 4). However the mutant receptor, despite being present in slightly greater quantities (Figure 5b), was unable to bind synthetic ARE as effectively as the WT (Figure 5a, compare lane 7 with 8 , and lane 9 with 10). Addition of excess unlabeled synthetic ARE reduced both WT and mutant signals, with the mutant band being less prominent than the WT (Figure $5 a$, lanes 5 and 6). The presence of nonspecific competitor DNA (ERE; and Oct2a oligonucleotide, the binding site for a ubiquitous transcription factor) did not reduce the band shifts, but mutant signal was still less intense than WT in both instances (Figure 5a, compare lanes 7 with 8 and lane 9 with 10 . The specificity of the band shift was confirmed by the absence of the most prominent band when radiolabeled ERE was used (Figure 5a, lanes 11 and 12). The ARE-binding deficit of the mutant ligand-AR complexes was quantified. In a first series of experiments using 2 different AREs, the mutant receptor had only $57.7 \%( \pm 5.17 \mathrm{SE})$ of the WT DNA-binding capacity (Figure $5 \mathrm{c}$, left). In a second series of experiments, a slightly modified technique was used to reduce background counts, and the DNA-binding defect was even more marked, with M886V displaying only $38 \%$ of the activity compared with normal controls (Figure 5 c, right). In comparison, 2 DNA binding-domain mutants, $\Delta$ Phe582 and $\Delta$ Arg615, displayed less than $20 \%$ of normal binding. In aggregate, the M886V AR had only about half the DNAbinding activity of the WT, and this defect in DNA binding was commensurate with the degree of impaired transactivation observed, as discussed earlier here.

The mutation affects TAD-LBD interaction. To determine whether the M886V mutation impairs interaction between the LBD and TAD, vectors encoding the $\mathrm{COOH}$-terminal LBD and the $\mathrm{NH}_{2}$-terminal TAD were constructed and expressed simultaneously in the presence of an ARE-driven reporter gene (Figure 6a). The LBD or TAD fragments by themselves did not display any androgen-inducible reporter gene activity. However, WT LBD fragment, when coexpressed with the TAD, resulted in an androgen-dependent increase in luciferase activity when exposed to physiological doses of DHT, indicating that AR fragments can interact to yield a functional protein (Figure 6a, left). Mutant LBD fragment, when coexpressed with $\mathrm{TAD}$, resulted consistently in a 22-25\% lower androgen-inducible activity compared with the WT LBD fragment in 3 independent experiments. Fusion proteins, comprising ARTAD fused to VP16AD, and ARLBD to the GAL4DBD, were coexpressed in HeLa cells, and protein-protein interactions were measured with a reporter vector containing multiple GAL4 DNA-binding sites in the mammalian twohybrid assay (Figure 6b). Interactions between TAD and LBD were specific and androgen dependent. Differences between WT and mutant were most evident when approximately equivalent quantities (50-100 ng) of LBD and TAD hybrid plasmids were cotransfected (Figure 6b, left). Mutant LBD fusion protein had only half the activity of the WT in this assay when exposed to subnanomolar doses of $\mathrm{MB}$ (Figure 6b, right). Thus, experiments with AR fragments using an ARE-driven reporter, and AR fusion proteins using GAL4-driven reporter, indicated that M886V impaired TAD-LBD interactions.

\section{Table 1}

Interactions of AR fusion proteins in the yeast two-hybrid assay

\begin{tabular}{cc}
\hline G(TAD)-AR(LBD) & G(TAD)-(AR) \\
G(DBD)-AR(LBD) & G(DBD)-AR(LBD)
\end{tabular}

$\begin{array}{ccc}\text { Experiment } & \text { WT } & \text { M886V } \\ 1 & 5.58 \pm 0.72 & 3.03 \pm 0.68 \\ 2 & 7.08 \pm 3.66 & 3.33 \pm 0.29 \\ 3 & 5.45 \pm 2.93 & 3.55 \pm 1.26 \\ \text { Mean } & 6.03 & 3.30\end{array}$

$\begin{array}{ccc}\text { Experiment } & \text { WT } & \text { M886V } \\ 1 & 244 \pm 21 & 51.4 \pm 4.5 \\ 2 & 379 \pm 193 & 197 \pm 87 \\ 3 & 154 \pm 51.4 & 124 \pm 43 \\ \text { Mean } & 259 & 124\end{array}$

Yeast GAL4-activation $[G(T A D)]$ and DNA-binding $[G(D B D)]$ domains were fused to full-length AR or LBD fragment to study interdomain interactions. Data (mean $\pm \mathrm{SE}$ ) represent the fold increase in $\beta$-galactosidase activity, in the presence and absence of $1 \mu \mathrm{M} \mathrm{MB}$, of 3 independent experiments, each in triplicate. 
a

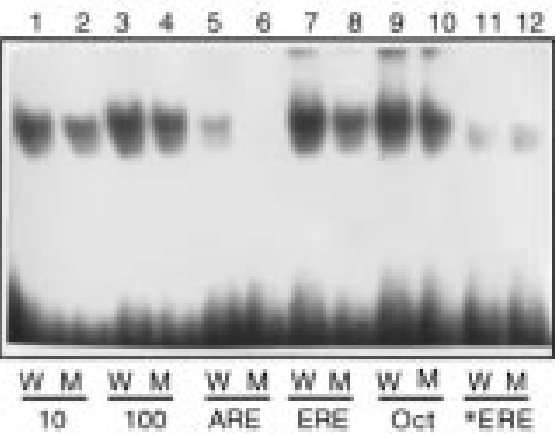

b

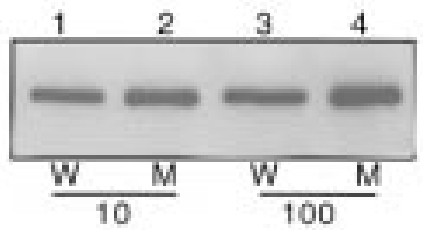

c

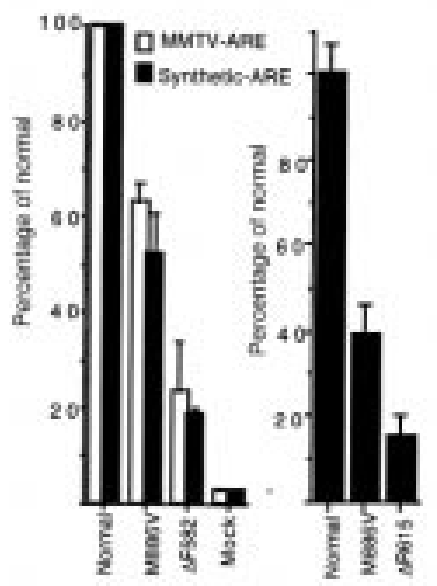

\section{Figure 5}

(a) DNA mobility gel shift assay. WT (W) or mutant (M) receptors were expressed in COS-7 cells and exposed to $10 \mathrm{nM}$ (lanes 1 and 2) or 100 $\mathrm{nM}$ (lanes 3-12) DHT. Equivalent quantities of immunoreactive AR from the cell extracts were added to binding reactions containing ${ }^{32} \mathrm{P}$-labeled synthetic ARE (lanes 1-10) or ERE (lanes 11 and 12) oligonucleotide sequences. Excess unlabeled ARE (lanes 5 and 6), ERE (lanes 7 and 8), or Oct (lanes 9 and 10) oligonucleotides were added as competitor DNA to demonstrate the specificity of the binding reaction. The dark band at the bottom represents unbound ${ }^{32}$ P-labeled DNA. (b) Immunoblot of WT or mutant receptors used in gel shift assay. Five microliters of representative cell extract (used in the gel shift assay depicted in Figure 5a) was exposed to either 10 or $100 \mathrm{nM}$ of DHT and was separated on an SDSPAGE gel. AR protein was identified with a specific antibody (PG-21). (c) Quantification of binding to AREs. Receptors were expressed in COS cells, exposed to $\left[{ }^{3} \mathrm{H}\right] \mathrm{MB}$ and equivalent quantities $(50,000 \mathrm{dpm})$ of $\left[{ }^{3} \mathrm{H}\right] \mathrm{MB}-\mathrm{AR}$ complexes incubated with $150 \mathrm{pmol}$ of either biotin-labeled natural ARE (an ARE from MMTV-LTR) or synthetic ARE in 2 independent series of experiments. Streptavidin-biotin-bound AREs were collected by centrifugation, and $\left[{ }^{3} \mathrm{H}\right] \mathrm{MB}$-labeled receptor bound to the AREs was quantified by scintillation counting. Known DNA binding-domain mutants $(\Delta \mathrm{F} 582, \Delta \mathrm{R} 615)$ with severe impairment of DNA binding were used for comparison. In the right panel, background counts were lowered by treating the lysate with dextran-coated charcoal and by centrifugation at 100,000 $\mathrm{g}$ for 1 hour. Assays using mock-transfected cells showed minimal background activity. Each data point was the mean of 2 experiments, and bars indicate their range.
The mutation affects $L B D-L B D$ interactions. To examine whether the M886V mutation can affect LBD-LBD interactions, we constructed hybrid expression vectors wherein full-length or truncated AR fragments were fused to the GAL4 DNA-binding [G(DBD)] or the GAL4-transactivation $[\mathrm{G}(\mathrm{TAD})]$ domains in the yeast two-hybrid assay (Table 1). No androgen-inducible activity was observed when GAL4-AR hybrid proteins were coexpressed with the GAL4 domains [G(DBD) or G(TAD)] alone (data not shown). Coexpressed G(TAD)-AR(LBD) and $\mathrm{G}(\mathrm{DBD})-\mathrm{AR}(\mathrm{LBD})$ hybrid proteins containing the WT LBD induced a 5- to 7-fold increase in transcriptional activity in the presence of androgen. In contrast, fusion proteins containing the mutant LBD were consistently only half as transcriptionally active as the WT in 3 independent experiments, each performed in triplicate. Defective interactions were also observed when a larger AR fragment, G(TAD)-AR(DBDLBD), was used (data not shown). Notably, when full-length AR [G(TAD)-(AR)] fusion protein was coexpressed, interactions were 2 orders of magnitude higher than with truncated AR recombinant proteins lacking ARTAD, indicating the importance of TAD-LBD interactions to the dimerization process. This increase in magnitude of interaction may reflect intramolecular LBD-TAD association (16) or the intermolecular effect of the TAD on the LBD (17). In all these experiments, M886V fusion proteins were consistently transcriptionally defective, having only about half the activity of the WT.

M886V disrupts interactions with the coactivator TIF2. Methionine 886 of the AR is close to residues known to interact with the steroid receptor coactivator TIF2 (18). We therefore examined the effect of $\mathrm{M} 886 \mathrm{~V}$ on coactivator function in 3 ways. First, we measured the effect of full-length TIF2 on WT and mutant AR transcriptional activity; second, the interaction of fusion proteins containing TIF2 and LBD; and third, the effect of full-length TIF2 on TAD-LBD interactions. Full-length TIF2 enhanced WT AR activity approximately 3-fold in a dosedependent manner (Figure 7a, left). However, M886V was defective and lowered coactivator function by $22-41 \%$. Significant impairment of TIF2 function was most prominent at a dose of $0.1 \mathrm{nM} \mathrm{MB}$ (Figure 7a, right). When fusion proteins containing GAL4DBDARLBD were coexpressed with VP16AD-TIF2, M886V caused a mean $37 \%$ lower receptor/coactivator interaction with doses of $\mathrm{MB}$ from 0.01 to $1 \mathrm{nM}$ (Figure $7 \mathrm{~b}$, left). Impaired interaction of TIF2 with M886V was not corrected by increasing the amount of TIF2 cDNA (Figure $7 \mathrm{~b}$, right). Defective interactions of mutant LBD with TIF2 were not due to differences in LBD fusion protein content, as measured by immunoblots and $\left[{ }^{3} \mathrm{H}\right] \mathrm{MB}$ binding (Figure $7 \mathrm{~b}$, bottom). To determine whether $\mathrm{NH}_{2}$ - and $\mathrm{COOH}$-terminal interactions of the AR were also regulated by coactivator, full-length TIF2 was coexpressed with WT or mutant GAL4DBD-ARLBD and VP16AD-ARTAD fusion proteins in the mammalian two-hybrid system. Interactions between the WT LBD and the TAD fusion proteins were increased up to 20 fold in the presence of TIF2. M886V disrupted this effect of TIF 2 by up to $28 \%$, consistent with the impaired TADLBD interactions observed previously (Figure 7c). 
a
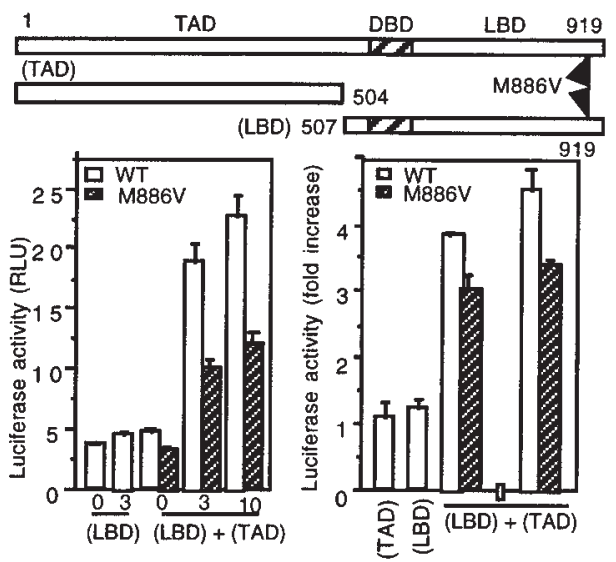

b

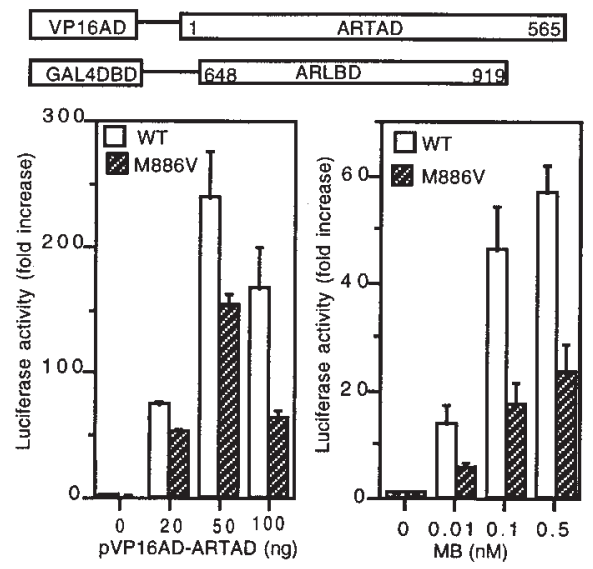

Figure 6

(a) Transcription activity of AR fragments. Deletion constructs encoding the ARTAD (amino acids 1-504) or the ARLBD (amino acids 507-919) were transiently transfected into CV-1 cells. Transcriptional activity (measured in RLU) of the WT or M886V LBD fragments, alone or coexpressed with TAD $(1 \mu \mathrm{g})$, was measured with a PMAM-LUC in the presence and absence of indicated amounts DHT (nM) (left). The experiments were repeated on another 2 occasions using independent plasmid preparations, and the results were expressed as fold increase in luciferase activity in the presence and absence of $3 \mathrm{nM} \mathrm{DHT}$ (right). (b) Interactions of LBD and TAD fusion proteins in the mammalian two-hybrid assay. The fusion proteins VP16AD-ARTAD and GAL4DBD-ARLBD were coexpressed in HeLa cells, and receptor TAD-LBD interactions were measured with $(17 \mathrm{~m})_{5^{-}}$ E1bTATA-Luc reporter plasmid. Cells were exposed to increasing doses of PVP16AD-ARTAD or MB as indicated. Data points represent mean \pm SE of at least 3 replicates and reflect fold increase in luciferase activity of cells exposed to MB over those not exposed to the androgen.

\section{Discussion}

Earlier studies $(2-4,19,20)$ have provided endocrine-biochemical evidence linking oligospermia and azoospermia, with or without other signs of undervirilization, to quantitative and/or qualitative abnormalities of the AR. There have been only 2 case reports $(6,21)$ of $A R$ point mutations in men with different degrees of impaired spermatogenesis. This study was undertaken to discover constitutional $A R$ mutations in a series of 173 men with varying degrees of impaired spermatogenesis. Among the subset of 84 men with severe, idiopathic oligospermia, we found 3, unrelated, each of whom had the same novel $A R$ variant: $M 886 \mathrm{~V}$. The fact that we did not find the variant among $400 \mathrm{control} A R$ alleles indi- cated that this was a pathogenic allelic variation. One of the men shaves infrequently; the other has Tanner grade 2 persistent postpubertal gynecomastia. These facts strengthened the idea that a cause-and-effect relationship might exist between the $A R$ variant and severe oligospermia. To prove its pathogenicity, we undertook the studies, the results of which are discussed later here.

The location of the Met886Val mutation in the LBD of the AR led us to expect some degree of androgen-binding abnormality because a nearby mutation, V889M, 3 residues downstream, has been reported to cause nearly complete androgen insensitivity due to defective androgen-binding capacity (22). Furthermore, all mutations so far described in exon 8 of the $A R$ manifest some abnormality of androgen binding (23). To our surprise, all androgen-binding properties of the M886V in genital skin fibroblasts of both probands were within normal limits. Similarly, the androgen-binding properties of the mutant AR were normal in 2 transfected mammalian cell lines. Thus, the M886V mutation, although residing in the hormone-binding domain of the AR, does not affect the conformation of the ligand-binding pocket of the LBD. However, the mutant AR was unable to transactivate normally and displayed only $50-70 \%$ of the transactivation ability of the WT in 3 different cell lines (COS, CV-1, HeLa), as measured by natural (MMTV-LTR) or multimeric ARE reporter genes. All transactivationdefective LBD mutations reviewed to date (23) have been associated with some form of androgen-binding abnormalities. In this regard, it is striking to note that the nearby V889M mutation caused nearly complete androgen insensitivity with increased androgen dissociation kinetics, despite normal equilibrium androgen-binding affinity (24). The activated mutant receptor had an impaired ability to bind oligonucleotides containing 2 different AREs: the first, an artificial ARE based on the consensus DNA-binding site for androgens; the second, MMTV-LTR, the naturally occurring steroid response element. Binding to either ARE was approximately $60 \%$ that of the WT. Thus, it is very likely that reduced ARE binding contributes to the reduced transactivation competence of the M886V AR. However, as discussed later, faulty intra- and intermolecular interactions of the mutant AR may, directly or indirectly, be contributory as well. Mutations such as M886V are particularly interesting, as they can illuminate functional subdomains that reside in the LBD.

Interactions between the $\mathrm{NH}_{2}$ - and $\mathrm{COOH}$-terminal domains of the AR have been observed using the mammalian two-hybrid system $(17,25)$, and a transcriptionally active complex forms when truncated proteins having only the $\mathrm{NH}_{2}$ - and $\mathrm{COOH}$-terminal domains of the AR are coexpressed in mammalian cells (16). Several lines of evidence indicate that defective TAD-LBD and LBDLBD interactions are important in the pathogenicity of M886V. M886 in the COOH-terminal region, directly or indirectly, interacts with the $\mathrm{NH}_{2}$-terminal portion of the AR. M886V impairs TAD-LBD interactions in both AR- and GAL4-driven reporter systems. M886V also consistently disrupted LBD-LBD interactions of GAL4-AR fusion proteins in the yeast two-hybrid assay. These data indicate that M886 has a role in interactions between the 
a

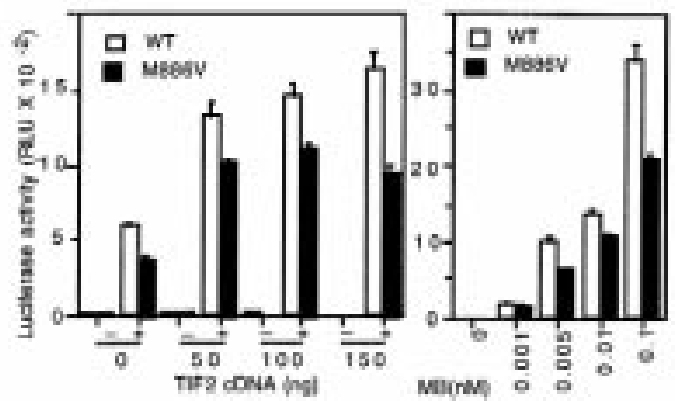

c

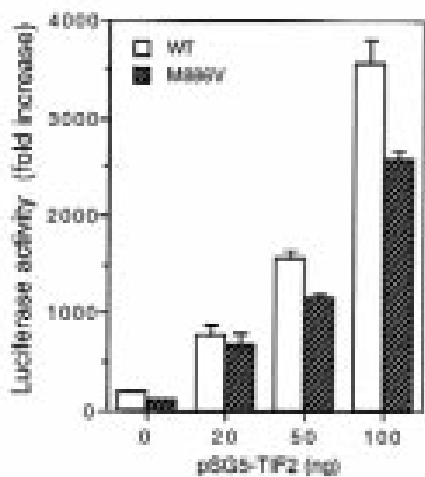

b

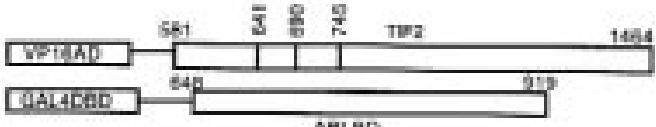

AHLAD
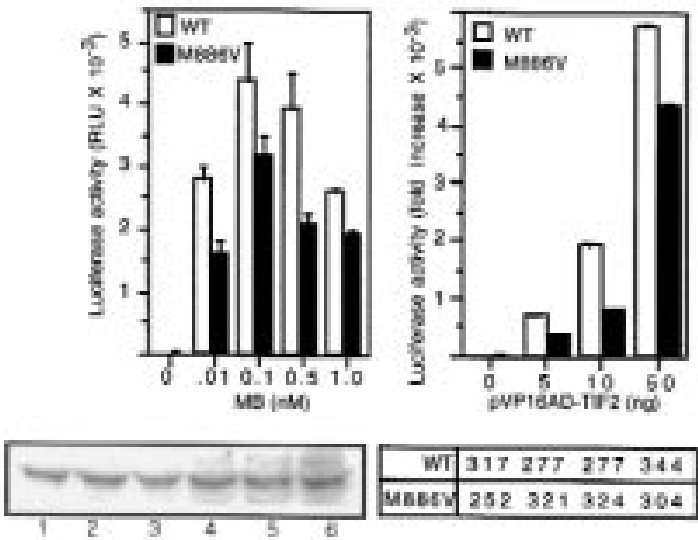

WT $317277 \quad 277344$

MEEST $252321324 \quad 304$

Figure 7

(a) Effect of TIF2 on AR activity in HeLa cells. Mutant or WT AR plasmids were cotransfected with the indicated amounts of cDNA encoding fulllength TIF2, with (+) or without (-) $0.01 \mathrm{nM} \mathrm{MB}$ (left). Cells were transfected with $50 \mathrm{ng}$ TIF2 cDNA and exposed to increasing doses of MB (right). AR activity was measured with a multimeric AR reporter gene (ARE-TATA-Luc). (b) Interactions of TIF2 and ARLBD fragments in the mammalian two-hybrid assay. The fusion proteins VP16AD-TIF2 and GAL4DBD-ARLBD were coexpressed in HeLa cells, and protein-protein interactions were measured with $(17 \mathrm{~m})_{5}$-E16TATA-Luc reporter plasmid. Amino acid positions of TIF2 and ARLBD fragments are numbered. Vertical bars within the TIF2 fragment indicate nuclear receptor-interacting box motifs ( $L X X L L)$, and numbers indicate the first $L$ of each consensus motif. In the left panel, cells were exposed to increasing doses of MB; in the right panel, cells were exposed to increasing doses of the VP16AD-TIF2 expression vector. Data are expressed as fold increase in luciferase activity with or without $1 \mathrm{nM} \mathrm{MB}$. Bottom panels show an immunoblot of WT (lanes 1-3) and mutant (lanes 4-6) GAL4DBB-ARLBD fusion proteins ( $\sim 51 \mathrm{kDa})$ from representative cell lysates (10 $\mu \mathrm{g}$ protein per lane) and specific [ $\left.{ }^{3} \mathrm{H}\right] \mathrm{MB}-\mathrm{binding}$ activity (fmol/mg protein) of cells transfected with WT or mutant GAL4DBB-ARLBD vector. (c) Effect ofTIF2 on TAD-LBD interactions in the mammalian two-hybrid assay. WT or mutant GAL4DBD-ARLBD fusion protein was coexpressed with VP16AD-ARTAD in HeLa cells, and TAD-LBD interactions were measured with a $(17 \mathrm{~m})_{5}-$ E1 16 TATA-Luc reporter plasmid (as in $\mathbf{b}$ ). The effect of cotransfecting increasing doses of a fourth vector, pSG5TIF2, encoding the full-length TIF2 protein, was measured as fold increase in luciferase activity with and without $0.1 \mathrm{nM}$ MB. Data were the mean \pm SE of at least 3 replicates.

functional domains of the AR. It is important to note that the nearby residue, V889, although a part of the ligand-binding pocket, also interacts with the TAD (26). Similarly, mutations of the human estrogen receptor affecting C530 (27), approximately 5 residues $\mathrm{NH}_{2}$-terminal to the homologue of AR M886, lose DNA-binding activity but retain normal estradiol affinity. Collectively, these data suggest strongly that a functional element centered around residue 886 of the AR has a role, not for ligand binding, but for interdomain interactions.

Recently, several steroid receptor coactivators (SRCs) have been characterized that mediate nuclear receptor interactions with the preinitiation complex and the chromatin template (28). The SRC gene family has several closely related homologues; of these, TIF2 has the greatest activity with respect to AR (29). Three highly conserved regions, each containing the nuclear receptor-interacting motif LXXLL, are located within the nuclear receptor-interacting domain of TIF2 (Figure 7b). The LXXLL motifs interact with core motifs in LBDs of nuclear receptors close to residue 886 of the AR (30). M886V significantly impairs TIF2 coactivator function in an AR-driven reporter system and also with chimeric proteins in the mammalian two-hybrid assay. In both systems, M886V reduces TIF2 coactivation function by approximately $40 \%$, equivalent to the defects in transactivation, DNA binding, and interdomain interactions observed. There is evidence that TIF2 interacts with both the TAD and LBD of steroid receptors, enhances the transcriptional activity of TAD and LBD separately, and has an additive effect when TAD and LBD are expressed simultaneously (31). Our data show that TIF2 increases by approximately 20 -fold the interactions between the $\mathrm{TAD}$ and $\mathrm{LBD}$ of the AR and that this interaction is disrupted by M886V (Figure 7c). Thus, it is not surprising that our mutation can affect interdomain interactions and DNA binding, as TAD-LBD interactions are thought to be critical for both functions (26). It is known that ligand-induced recruitment of an LXXLL motif of SRC-1 to the retinoic acid receptor (RAR) promotes heterodimerization to the retinoic acid $\mathrm{X}$ receptor (RXR) and binding of a second LXXLL motif molecule to RXR. The 2 adjacent LXXLL motifs in SRC-1 contact homologous residues in the LBDs of the RXR-RAR dimers, thereby forming a 
charge clamp to stabilize the dimerization complex (32). Our findings that the M886V mutation affects many AR processes, including TAD-LBD and LBD-LBD interactions, DNA binding, and transactivation competence, are consistent with the integrative role that coactivators like TIF2 may have in these post-ligand-binding events.

Because a nearby $A R$ mutation (E897Q) also preserves ligand binding while disrupting TAD-LBD and LBD-TIF2 interactions (18), our data suggest that M886 contributes to a functional subdomain of the AR LBD that does not mediate androgen binding but is important for interaction with TIF2. This functional dichotomy in the AR LBD is not implausible considering that intermolecular interactions depend on surface properties, whereas ligand-binding pockets are buried within the hydrophobic cores of all steroid receptor LBDs crystallized to date (33). The identification of other transactivation-defective LBD mutations $(6,21)$ that do not affect ligand binding suggests the possibility that disruption of one or another coactivator activity may be a common contributor to pathogenesis of severe oligospermia and male infertility. Given that at least one of these abnormalities is correctable by hormonal manipulation $(6,21)$, understanding the pathogenetic mechanisms may lead to effective therapeutic strategies.

\section{Acknowledgments}

We thank G. Jenster (Anderson Cancer Center, Houston, Texas, USA) for his kind gift of ARE-TATA-Luc, G. Prins (University of Illinois, Chicago, Illinois, USA) for the AR antibody PG-21, and P. Chambon (IGBMC, Strasbourg, France) for PSG5-TIF2. This work is supported by grants from the Fonds de la Recherche en Santé du Québec (Hydro-Québec), Fonds pour la Formulation de Chercheurs et l'Aide a la Recherche, and the Medical Research Councils of Canada and Singapore. A.A.R. Abdullah is grateful for personal support from the University of Bahrain.

1. World Health Organization. 1991. Infertility: a tabulation of available data on the prevalence of primary and secondary infertility. WHO. Geneva, Switzerland. 1-72.

2. Aiman, J., and Griffin, J.E. 1982. The frequency of androgen receptor deficiency in infertile men. J. Clin. Endocrinol. Metab. 54:725-732.

3. Bouchard, P., et al. 1986. Androgen insensitivity in oligospermic men: a reappraisal. J. Clin. Endocrinol. Metab. 63:1242-1246.

4. Morrow, A.F., et al. 1987. Variable androgen receptor levels in infertile men. J. Clin. Endocrinol. Metab. 64:1115-1121.

5. Grino, P.B., Griffin, J.E., Cushard, W.G., and Wilson J.D. 1988. A mutation of the androgen receptor associated with partial androgen resistance, familial gynaecomastia, and fertility. J. Clin. Endocrinol. Metab. 66:754-761.

6. Wang, Q., et al. 1998. Azoospermia associated with a mutation in the ligandbinding domain of an androgen receptor displaying normal ligand binding, but defective transactivation. J. Clin. Endocrinol. Metab. 83:4303-4309.

7. Tsukada, T., Inoue, M., Tachibana, S., Nakai Y., and Takebe, H. 1994. An androgen receptor mutation causing androgen resistance in the undervirilised male syndrome. J. Clin. Endocrinol. Metab. 79:1202-1207.

8. World Health Organization. 1992. Laboratory manual for the examination of human semen and sperm-cervical mucus interactions. 3rd edition. R.J. Aitken et al., editors. WHO Special Program of Research, Development, and Research Training on Human Reproduction. Geneva, Switzerland. 1-107.

9. Yong, E.L., Chua, K.L., Yang, M., Roy, A., and Ratnam, S.S. 1994. Complete androgen insensitivity due to a splice-site mutation in the androgen receptor gene and genetic screening with single stranded conformation polymorphism (SSCP). Fertil. Steril. 61:856-862.
10. Gottlieb, B., Kaufman, M., Pinsky, L., Leboeuf, G., and Sotos, J.F. 1987. Extracellular correction of the androgen-receptor transformation defect in two families with complete androgen resistance. J. Steroid Biochem. 28:279-284

11. Tut, T.G., Ghadessy, F., Trifiro, M.A., Pinsky, L., and Yong, E.L. 1997. Polyglutamine tracts in the androgen receptor are associated with reduced transactivation, defective sperm production and male infertility. J. Clin. Endocrinol. Metab. 82:3777-3782.

12. Prins, G.S., Birch, L., and Greene, G.L. 1991. Androgen receptor localization in different cell types of the adult rat prostate. Endocrinology. 129:3187-3199.

13. Roche, P.J., Hoare, S.A., and Parker, M.G. 1992. A consensus DNA binding site for the androgen receptor. Mol. Endocrinol. 6:2229-2235.

14. Yu, V.C., et al. 1991. RXRb: a coregulator that enhances binding of retinoic acid, thyroid hormone, and vitamin $\mathrm{D}$ receptors to their cognate response elements. Cell. 67:1251-1266.

15. Glass, C.K., et al. 1987. A c-erb-A site in rat growth hormone gene mediates transactivation by thyroid hormone. Nature. 329:738-741.

16. Doesburg, P., et al. 1997 Functional in vivo interaction between the amino-terminal, transactivation domain and the ligand binding domain of the androgen receptor. Biochemistry. 36:1052-1064.

17. Langley, E., Zhou, Z.X., and Wilson, E.M. 1995. Evidence for an anti-parallel orientation of the ligand-activated human androgen receptor dimer. J. Biol. Chem. 270:29983-29990.

18. Berrevoets, C.A., Doesburg, P., Steketee, K., Trapman, J., and Brinkman, A.O. 1998. Functional interactions of the AF-2 activation domain core region of the human androgen receptor with the amino-terminal domain and with the transcriptional coactivator TIF2 (transcriptional intermediary factor 2). Mol. Endocrinol. 12:1172-1183.

19. Migeon, C.J., et al. 1984. A clinical syndrome of mild androgen insensitivity. J. Clin. Endocrinol. Metab. 59:672-678.

20. Smallridge, R.C., et al. 1984. Androgen receptor abnormalities in identical twins with oligospermia. Am. J. Med. 77:1049-1054.

21. Yong, E.L., Ng, S.C., Roy, A.C., Yun, G., and Ratnam, S.S. 1994. Pregnancy after hormonal correction of severe spermatogenic defect due to mutation in androgen receptor gene. Lancet. 344:826-827.

22. De Bellis, A., et al. 1994. Characterisation of mutant androgen receptors causing partial androgen insensitivity syndrome. J. Clin. Endocrinol. Metab. 78:513-522.

23. Gottlieb, B., et al. 1998. The Androgen Receptor Gene Mutations Database. Nucleic Acids Res. 26:234-238.

24. Zhou, Z.X., Lane, M.V., Kemppainen, J.A., French, F.S., and Wilson, E.M. 1995. Specificity of ligand-dependent androgen receptor stabilization: receptor domain interactions influence ligand dissociation and receptor stability. Mol. Endocrinol. 9:208-218.

25. Ikonen, T., Palvimo, J.J., and Janne, O.A. 1997. Interaction between the amino- and carboxyl-terminal regions of the rat androgen receptor modulates transcriptional activity and is influenced by nuclear receptor coactivators. J. Biol. Chem. 272:29821-29828.

26. Langley, E., Kemppainen, J.A., and Wilson, E.M. 1998. Intermolecular $\mathrm{NH} 2$-/carboxyl-terminal interactions in androgen receptor dimerization revealed by mutations that cause androgen insensitivity. J. Biol. Chem. 273:92-101.

27. Neff, S., Sadowski, C., and Miksicek, R.J. 1994. Mutational analysis of cysteine residues within the hormone-binding domain of the human estrogen receptor identifies mutants that are defective in both DNAbinding and subcellular distribution. Mol. Endocrinol. 8:1215-1223.

28. Voegel, J.J., et al. 1998. The coactivator TIF2 contains three nuclear receptor-binding motifs and mediates transactivation through CBP binding-dependent and -independent pathways. EMBOJ. 17:507-519.

29. Xiu, F.D., et al. 1998. Nuclear receptor binding sites of coactivators glucocorticoid receptor interacting protein (GRIP1) and steroid receptor coactivator 1 (SRC-1): multiple motifs with different binding specificities. Mol. Endocrinol. 12:302-313.

30. Nolte, R.T., et al. 1998. Ligand binding and co-activator assembly of the peroxisome proliferator-activated receptor-gamma. Nature. 395:137-143.

31. Onate, S.A., et al. 1998. The steroid receptor coactivator-1 contains multiple receptor interacting and activation domains that cooperatively enhance the activation function 1 (AF1) and AF2 domains of steroid receptors. J. Biol. Chem. 273:12101-12108.

32. Westin, S., et al. 1998. Interactions controlling the assembly of nuclearreceptor heterodimers and co-activators. Nature. 395:199-202.

33. Tanenbaum, D.M., Wang, Y., Williams, S.P., and Sigler, P.B. 1998. Crystallographic comparison of the estrogen and progesterone receptor's ligand binding domains. Proc. Natl. Acad. Sci. USA. 95:5998-6003. 